\title{
Validation of Microcirculatory Parameters Derived from the Standard Two-Compartment Model with Murine Xenografts Model
}

\author{
Septian Hartono, ${ }^{1,2}$ Choon Hua Thng, ${ }^{1}$ Richard Weijie Ong, ${ }^{3}$ Quan Sing Ng, \\ Tony Kiat Hon Lim, ${ }^{5}$ Sidney Wing Kwong Yu, ${ }^{6}$ The Hung Huynh, ${ }^{3}$ \\ Laurent Martarello, ${ }^{7}$ and Tong San Koh ${ }^{1,2}$ \\ ${ }^{1}$ Department of Oncologic Imaging, National Cancer Centre, Singapore 169610 \\ ${ }^{2}$ School of Electrical and Electronic Engineering, Nanyang Technological University, Singapore 639798 \\ ${ }^{3}$ Laboratory of Molecular Endocrinology, National Cancer Centre, Singapore 169610 \\ ${ }^{4}$ Department of Medical Oncology, National Cancer Centre, Singapore 169610 \\ ${ }^{5}$ Department of Pathology, Singapore General Hospital, Singapore 169608 \\ ${ }^{6}$ Department of Nuclear Medicine, Singapore General Hospital, Singapore 169608 \\ ${ }^{7}$ Roche-Singapore Translational Medicine Hub, Singapore 237994
}

Correspondence should be addressed to Septian Hartono; septian.hartono@nccs.com.sg

Received 19 June 2014; Revised 23 December 2014; Accepted 24 December 2014

Academic Editor: Takahiro Yamauchi

Copyright $\odot 2015$ Septian Hartono et al. This is an open access article distributed under the Creative Commons Attribution License, which permits unrestricted use, distribution, and reproduction in any medium, provided the original work is properly cited.

The purpose of this study was to validate DCE-MRI parameters such as blood flow $(F)$, permeability surface area product (PS), fractional intravascular space $\left(v_{1}\right)$, and fractional extracellular extravascular space $\left(v_{2}\right)$, obtained using a standard two-compartment model against other established analysis methods and histological indices. DCE-MRI datasets of 28 mice implanted with various human cancer xenografts were acquired and analyzed. Statistically significant correlations were found between the parameters derived from the standard two-compartment model $\left(v_{1}, v_{2}, F\right.$, and PS) with the histological markers of intravascular and interstitial space and with the corresponding flow and permeability estimates obtained by the initial slope method and Patlak plot, respectively.

\section{Introduction}

It is important to validate the parameters derived from DCEMRI against established techniques and to ascertain that the DCE-MRI parameters reflect the actual microcirculatory state and pathophysiology of the tumors imaged. Previous DCE-MRI validation studies [1-4] have attempted to correlate Generalized Kinetic (GK) model parameters, namely, the transfer constant $K^{\text {trans }}$ and the fractional interstitial volume $v_{e}$, with histological indices of the tumor microvasculature. However, according to GK model theory, $K^{\text {trans }}$ incorporates both the effects of blood flow and vessel permeability [5] and a recent simulation study [6] has shown that $K^{\text {trans }}$ has a significant positive correlation with blood flow, permeability, and blood volume, as well as a significant negative correlation with interstitial volume. With such ambiguities in interpretation, it might be difficult to identify appropriate markers for validation of $K^{\text {trans }}$.

The standard two-compartment model [7-10] describes tissue microcirculation with distinct parameters, namely, blood flow $(F)$, vessel permeability surface area product (PS), fractional vascular volume $\left(v_{1}\right)$, and fractional interstitial volume $\left(v_{2}\right)$. These physiological parameters can be readily validated by comparison with appropriate histological markers or other established tracer techniques. More complex tracer kinetic models such as the distributed parameter models [10] are also capable of separately estimating blood flow and permeability. However, the distributed parameter 
TABLE 1: Study population.

\begin{tabular}{lc}
\hline Type of xenograft & Number \\
\hline Hepatocellular carcinoma (HCC) & 7 \\
Gastric/stomach cancer (GC) & 5 \\
Lung cancer (LC) & 4 \\
Renal cell carcinoma (RCC) & 3 \\
Gastrointestinal stromal tumor (GIST) & 3 \\
Colorectal cancer (COL) & 3 \\
Pancreatic cancer (PAN) & 2 \\
Squamous cell carcinoma (SCC) & 1 \\
\hline
\end{tabular}

models require rapid sampling of tissue tracer concentration curves and might not be suitable for use in the preclinical setting where the temporal resolution of the DCE-MRI scans is low compared to the rapid murine circulation.

In this study, we attempt to validate parameters of the standard two-compartment model derived from DCE-MRI of human cancer xenografts in mice by comparing $v_{1}$ and $v_{2}$ with histological markers; and by comparing $F$ and PS with the corresponding estimates obtained using a macromolecular tracer (Galbumin) with established tracer analysis techniques, namely, the initial slope method and Patlak plot [11].

\section{Materials and Methods}

2.1. Mice and Tumor Cell Line. This study was approved by the Institutional Animal Care and Use Committee and all mice were maintained according to the Guide for the Care and Use of Laboratory Animals published by the National Institutes of Health, USA. Male nonobese diabetic (NOD) mice $(n=$ 28, 9-10 weeks old) with severe combined immunodeficiency (SCID, Animal Resources Centre, Canning Vale, Western Australia) were used as host animals for various types of human cancer xenografts. Details of the xenograft model and the formation of cancer cell lines can be found in [12]. Eight different types of human xenografts (Table 1) were implanted subcutaneously in the SCID mice. These tumors are known to differ in degree of angiogenesis.

2.2. DCE-MRI Protocol. DCE-MRI experiments were performed on a 7-T MR scanner (Bruker ClinScan, Bruker BioSpin MRI GmbH, Ettlingen, Germany) using a threedimensional (3D) spoiled gradient recalled sequence (FLASH $3 \mathrm{D})$ with the following parameters: repetition time $\mathrm{TR}=$ $3.04 \mathrm{~ms}$, echo time $\mathrm{TE}=1.23 \mathrm{~ms}$, field-of-view $\mathrm{FOV}=36 \times$ $36 \mathrm{~mm}^{2}, 128 \times 128$ matrix half-Fourier reconstructed to 256 $\times 256,8 \times 1 \mathrm{~mm}^{2}$ slices, final image resolution of $0.14 \times 0.14 \times$ $1 \mathrm{~mm}^{2}$, and acquisition time of $2 \mathrm{~s}$.

Tissue precontrast and postcontrast $T_{1}$ values were estimated using the dual flip angle technique $[13,14]$. To estimate the tissue native $T_{1}$ values, precontrast images were acquired with flip angles of $6^{\circ}$ and $14^{\circ}$. For each flip angle, 5 repetitions were performed and averaged. One hundred and thirty postcontrast acquisitions were performed with flip angle $14^{\circ}$ over a period of $260 \mathrm{s.} 50 \mu \mathrm{L}$ of gadoterate meglumine, INN (Dotarem, Guerbet S.A., Villepinte, France), was injected manually through the tail vein at the first postcontrast acquisition and was followed by a $50 \mu \mathrm{L}$ saline flush.

2.3. Phantom Validation. At low concentrations, contrast agent concentration $C$ can be estimated by the difference in longitudinal relaxation rates

$$
r_{1} C=\frac{1}{T_{1}^{C}}-\frac{1}{T_{1}^{0}}
$$

where $T_{1}^{C}$ and $T_{1}^{0}$ are the postcontrast and precontrast (native) $T_{1}$ values, respectively, and $r_{1}$ denotes the longitudinal relaxivity. A phantom study was performed to validate the $T_{1}$ values estimated by the above variable flip angle protocol by comparing with those derived from inversion recovery measurements (inversion time $\mathrm{TI}=50,100,200$, $400,600,800,1000,1500,2000,4000 \mathrm{~ms}$; repetition time TR $=5000 \mathrm{~ms}$ ). The phantom consists of tubes of saline diluted with $0.05 \mathrm{~g} / \mathrm{L}$ of copper sulphate to mimic $T_{1}$ value in human body tissue at $1.5 \mathrm{sec}$ and filled with various concentration of Dotarem at 0.01, 0.05, and 0.1 mM.

2.4. Galbumin-Enhanced Imaging Protocol. Galbumin (BioPal, USA) is gadolinium-labeled bovine albumin with a molecular weight of $\sim 74 \mathrm{kDa}$. Galbumin-enhanced scans commenced $15 \mathrm{~min}$ after the Gd-DOTA-enhanced scans, when the tissue enhancement due to Gd-DOTA has visibly faded. The Galbumin dynamic imaging protocol consists of two phases: an initial rapid imaging phase consisting of 15 acquisitions at $2 \mathrm{~s}$ temporal resolution using the $3 \mathrm{D}$ VIBE sequence mentioned above and a delayed phase of 15 acquisitions, with $58 \mathrm{~s}$ delay between each acquisition. A dose of $0.1 \mathrm{~mL}$ of Galbumin at $25 \mathrm{mg} / \mathrm{mL}$ was manually injected through the tail vein after the first set of dynamic images and followed by a $50 \mu \mathrm{L}$ saline flush.

2.5. Data Processing. Postprocessing was performed offline on an Intel Core 2 Duo personal computer with Matlab (MathWorks, Natick, MA). For reduced inflow effects and wrap, only the 4 central slices from the imaging volume (of 8 slices) were selected for processing. Regions-of-interest (ROIs) corresponding to the lesions were manually outlined on the 4 central slices.

Galbumin concentration was estimated from the Galbumin-enhanced dynamic scans by the change in longitudinal relaxation rates after injection of Galbumin.

2.6. Tracer Kinetic Modeling. Consider a bicompartmental tissue system where the first compartment represents the vascular space and the second compartment represents the interstitial space. Assuming well-mixed compartments, 
tracer concentration in each compartment at time $t$ is given as follows $[7,8]$ :

$$
\begin{aligned}
v_{1} \frac{d}{d t} C_{1}(t)= & F \rho\left(C_{\text {in }}(t)-C_{1}(t)\right) \\
& -\operatorname{PS} \rho\left(C_{1}(t)-C_{2}(t)\right), \\
v_{2} \frac{d}{d t} C_{2}(t)= & \operatorname{PS} \rho\left(C_{1}(t)-C_{2}(t)\right),
\end{aligned}
$$

where $F$ denotes blood flow, $C_{1}$ and $C_{2}$ denote tracer concentration in the vascular and interstitial space, respectively, PS denotes the permeability surface area product, and $\rho$ denotes the tissue of density, which is set at $1 \mathrm{~g} / \mathrm{mL} . v_{1}$ is the fractional vascular volume, $v_{2}$ is the fractional interstitial volume, and $C_{\text {in }}(t)$ denotes the arterial input concentration.

The operational equation for analysis of the DCE imaging data can be expressed as

$$
C_{\text {tiss }}(t)=F C_{\text {in }}(t) \bigotimes R(t),
$$

where $C_{\text {tiss }}$ denotes the tracer concentration in the tissue voxel and $\otimes$ denotes the convolution operator. $R(t)$ is the impulse residue response function, which describes the fractional amount of tracer remaining in the tissue owing to an impulse input at time $t$. And $R(t)$ is given by

$$
R(t)=A \exp \left(s_{1} t\right)+(1-A) \exp \left(s_{2} t\right)
$$

where $s_{1}$ and $s_{2}$ are the solutions of the following quadratic equation [15]:

$$
\begin{aligned}
& s^{2}+\left(\frac{\mathrm{PS} \rho}{v_{1}}+\frac{\mathrm{PS} \rho}{v_{2}}+\frac{F \rho}{v_{1}}\right) s+\frac{\mathrm{PS} \rho}{v_{2}} \frac{F \rho}{v_{1}}=0, \\
& A=\frac{s_{1}+\left(\mathrm{PS} \rho / v_{1}\right)+\left(\mathrm{PS} \rho / v_{2}\right)}{s_{1}-s_{2}} .
\end{aligned}
$$

2.7. Galbumin Kinetic Analysis. The Galbumin-enhanced scans were analyzed using more established methods to derive alternative estimates for blood flow and vessel permeability. Galbumin concentration-time data $C_{\text {tiss }}^{\mathrm{Gal}}(t)$ corresponding to the first rapid imaging phase was analyzed using the maximal slope method [16] to yield estimates for blood flow

$$
F_{\max }=\frac{\left.(d / d t) C_{\mathrm{tiss}}^{\mathrm{Gal}}(t)\right|_{\max }}{\left.C_{A}^{\mathrm{Gal}}(t)\right|_{\max }},
$$

where $C_{A}^{\mathrm{Gal}}(t)$ is the arterial input function sampled from the Galbumin-enhanced scans.

Galbumin concentration-time data for the delayed imaging phase was analyzed using the Patlak plot method to derive the Galbumin influx rate constant $K_{i}$, which is indicative of vessel permeability [17]:

$$
\frac{C_{\mathrm{tiss}}^{\mathrm{Gal}}(t)}{C_{A}^{\mathrm{Gal}}(t)}=K_{i} \frac{\int_{0}^{t} C_{A}^{\mathrm{Gal}}(\tau) d \tau}{C_{A}^{\mathrm{Gal}}(t)}+v_{0},
$$

where $v_{0}$ is the ordinate intercept of the Patlak plot.

\subsection{Histopathology}

2.8.1. Tissue Sectioning. The tumor was manually dissected from the subcutaneous tissue. It was orientated in a plane similar to the imaging plane. The superior, inferior, left, and right borders were stained with different colors to allow orientation. The tumor was manually sectioned and subsequently fixed and embedded. A microtome section was obtained from the superior aspect of each section.

2.8.2. CD-31 Stain. Tumor cryosections ( $5 \mu \mathrm{m}$ thick) were pretreated using optimized antigen retrieval methods and then immunohistochemical staining was performed. Vasculature was stained using a platelet/endothelial cell adhesion molecule/CD31 antibody and CD34 antibody (1:100 dilution; BD PharMingen, San Diego, CA). Visualization was done with automated stainer and biotin-avidin complex. The percentage of area stained for CD31 is used as a measure of the intravascular space.

2.8.3. Masson's Trichrome. Interstitial collagen tissue was used as a marker of the interstitial space and was stained with the Masson's trichrome stain. The digital image was threshold for interstitial collagen. The percentage of area stained for collagen is used as a measure of the interstitial space.

2.9. Microscope/Image Acquisition. Digital images of the tumor slices were created at a magnification of $\times 400$ with a Nikon microscope (Nikon Instruments, Melville, NY). Image analysis for vessel counting was done using Nikon imaging software (NIS-Elements Basic Research 3.0, Nikon Instruments, Melville, NY). The software allowed the application of standardized computational algorithms as well as review and refinement of the results of those algorithms by human operators [18]. A camera resolution of $1300 \times$ 1028 pixels would result in a pixel size of $0.240 \mu \mathrm{m} /$ pixel. The pixel size of the DCE-MRI image was calculated by dividing the field-of-view with the resolution of the matrix. A grid sized to the DCE-MRI pixel is overlaid onto the digital histopathology image. Subsequent analysis (such as vessel count or percentage area of staining) is calculated with respect to each grid box (which correspond in size to the DCE-MRI pixel).

2.10. Statistical Analysis. Three hotspot areas were chosen in each tumor and the median value of each ROI was taken as its representative value. Histological sections were orientated according to the DCE-MRI images so that they will correspond to the same plane.

Correlation analysis using Pearson correlation coefficient was performed to compare the parameters derived from the standard two-compartment model $\left(v_{1}, v_{2}, F\right.$, and PS) with the histological markers of intravascular and interstitial space and with the corresponding flow and permeability estimates obtained by the initial slope method and Patlak plot, respectively. All statistical analyses were performed using STATA v. 10 (StataCorp LP, TX), assuming a two-sided test at the conventional 0.05 level of significance. 


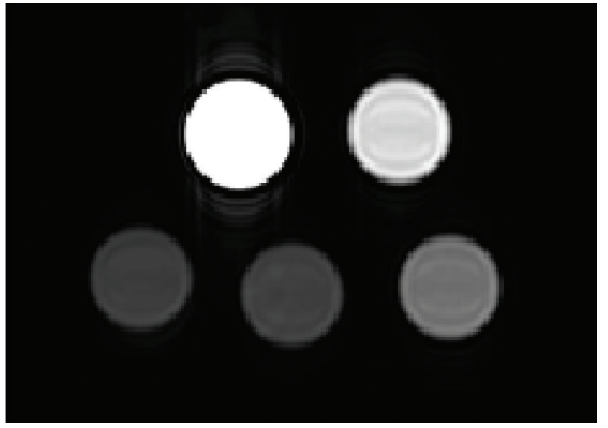

(a)

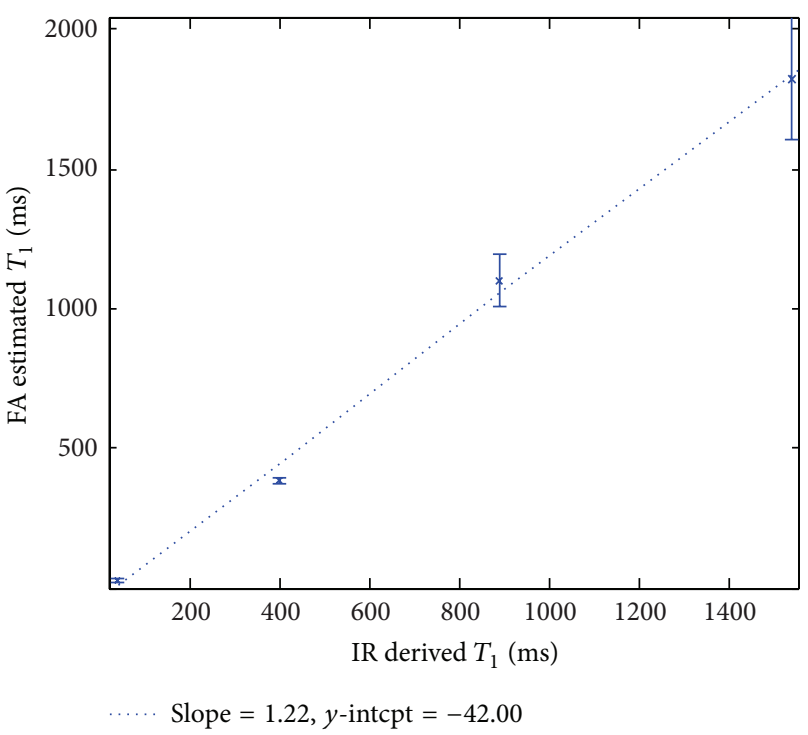

(b)

Figure 1: (a) Phantom setup, which consists of tubes of saline diluted with $0.05 \mathrm{~g} / \mathrm{L}$ of copper sulphate to mimic $T_{1}$ value in human body tissue at $1.5 \mathrm{sec}$ and filled with various concentration of Dotarem; (b) comparison between $T_{1}$ derived from the inversion recovery sequence with $T_{1}$ estimated by dual flip angle method.

TABLE 2: Comparison of DCE-MRI parameters in clinical and preclinical settings.

\begin{tabular}{lcc}
\hline Parameters & Human & Animal \\
\hline Machine & Avanto (1.5T, Siemens) & ClinScan (7T, Bruker) \\
Platform & syngoMR & syngoMR \\
Sequence & FLASH 3D & FLASH 3D VIBE \\
FoV & $40 \times 40 \mathrm{~cm}$ & $36 \times 36 \mathrm{~mm}$ \\
TR & $3.03 \mathrm{~ms}$ & $3.04 \mathrm{~ms}$ \\
TE & $1.17 \mathrm{~ms}$ & $1.23 \mathrm{~ms}$ \\
Temp. resolution & $1.38 \mathrm{~s}$ & $2 \mathrm{~s}$ \\
Slice & 6 & 8 \\
Slice thickness & $8 \mathrm{~mm}$ & $1 \mathrm{~mm}$ \\
Base resolution & $256 \times 256$ & $128 \times 128$ \\
Flip angle & $6^{\circ} \& 18^{\circ}$ & $6^{\circ} \& 14^{\circ}$ \\
\hline
\end{tabular}

\section{Results}

Parameters of DCE-MRI sequences used in human studies and its translated version in animal studies were given in Table 2. The sequences were based on a similar basic FLASH sequence. The values of TR and TE were set to be as similar as possible within the allowances given by the machine.

Comparison between $T_{1}$ values derived by the inversion recovery sequence with those estimated by the dual flip angle method was given in Figure 1. A linear correlation with slope of 1.22 and negligible ordinate intercept was obtained. Hence a reasonable precision of $T_{1}$ values estimated by the latter method was acquired and indicated the reliability of the estimated concentration values in the mouse tissue.

The various microcirculatory parameters estimated by the standard two-compartment model for each type of xenograft are presented in Table 3. Blood flow $F$ is highest in the renal xenografts, reaching $164.15 \pm 1.05 \mathrm{~mL} / 100 \mathrm{~mL} / \mathrm{min}$, and lowest in the HCC, GIST, and colorectal xenografts at around 25-30 $\mathrm{mL} / 100 \mathrm{~mL} / \mathrm{min}$.

Correlations between the microcirculatory parameters derived by the standard two-compartment model with their respective immunohistochemistry staining were presented in Figure 2. Statistically significant correlations $(P<0.001)$ were found for each parameter.

An example of a hepatocellular carcinoma xenograft is shown in Figure 3. Histological sections of the tumor with CD31 and Masson's trichrome staining are shown in Figures $3(\mathrm{~b})$ and 3(e). The hotspots of each staining are shown in Figures 3(c) and 3(f). The blood vessels and the interstitial collagen tissue are outlined in light green in the hotspot. The percentage of area of blood vessels is $3.3 \%$ and the interstitial volume is $18.55 \%$. The values are similar to those obtained with DCE-MRI, as shown in Figures 3(a) and 3(d), with percentage of blood volume measured at $3.499 \%$ and interstitial volume at $18.435 \%$.

The maps of maximum slope $F_{\max }$ and influx rate constant $K_{i}$ derived from Galbumin-enhanced scans are shown in Figures 4(b) and 4(d). The maps of blood flow and permeability derived from DCE-MRI are shown in Figures 4(a) and 4(c).

\section{Discussion}

Validation studies of plasma flow in DCE-CT have been performed using microspheres. Stewart et al. [19] derived the hepatic arterial blood flow $\left(\mathrm{H}_{\mathrm{A}} \mathrm{BF}\right)$ from a dual-input version of the adiabatic tissue homogeneity model and compared it with the result obtained from radiolabeled microsphere studies. A strong correlation was observed between $\mathrm{H}_{\mathrm{A}} \mathrm{BF}$ values from both techniques, with $r^{2}=0.81 \pm 0.05(P<0.05)$. 
TABLE 3: Microcirculatory parameters (mean \pm standard deviation) estimated by the standard two-compartment model for each type of xenograft.

\begin{tabular}{lcccc}
\hline Type of xenograft & $F(\mathrm{ml} / \mathrm{min} / 100 \mathrm{~mL})$ & PS $(\mathrm{ml} / \mathrm{min} / 100 \mathrm{~mL})$ & $v_{1}(\%)$ & $0.89 \pm 0.44$ \\
\hline HCC & $28.73 \pm 6.74$ & $3.98 \pm 2.00$ & $1.70 \pm 0.86$ & $7.18 \pm 2.75$ \\
Gastric & $60.59 \pm 30.32$ & $4.22 \pm 1.50$ & $4.06 \pm 1.03$ & $17.29 \pm 4.06$ \\
Lung & $51.25 \pm 18.99$ & $6.00 \pm 2.43$ & $4.56 \pm 0.08$ & $10.87 \pm 7.95$ \\
RCC & $164.15 \pm 1.05$ & $6.27 \pm 4.17$ & $0.82 \pm 2.28$ & $22.92 \pm 2.28$ \\
GIST & $26.83 \pm 4.01$ & $3.07 \pm 1.13$ & $2.79 \pm 1.60$ & $14.78 \pm 17.81$ \\
Colorectal & $27.86 \pm 9.29$ & $3.68 \pm 1.61$ & $2.02 \pm 0.05$ & $12.80 \pm 5.84$ \\
Pancreas & $34.64 \pm 4.00$ & $7.39 \pm 0.21$ & 5.18 & 22.75 \\
SCC & 98.99 & 11.8 & & \\
\hline
\end{tabular}

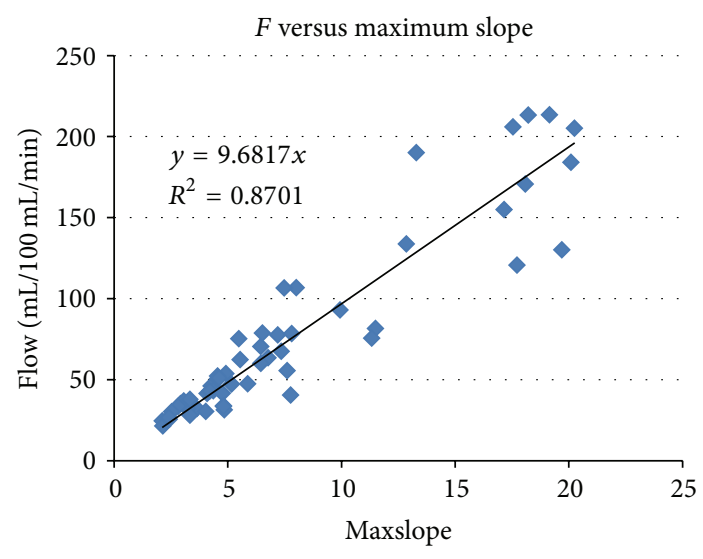

(a)

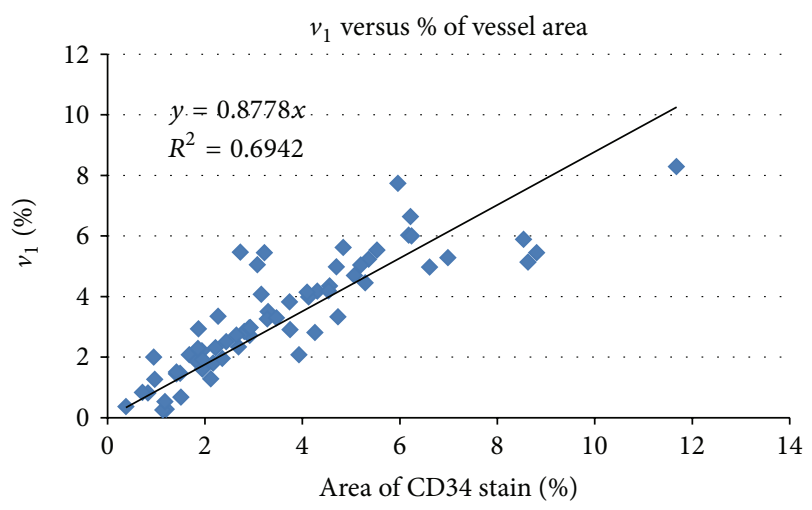

(c)

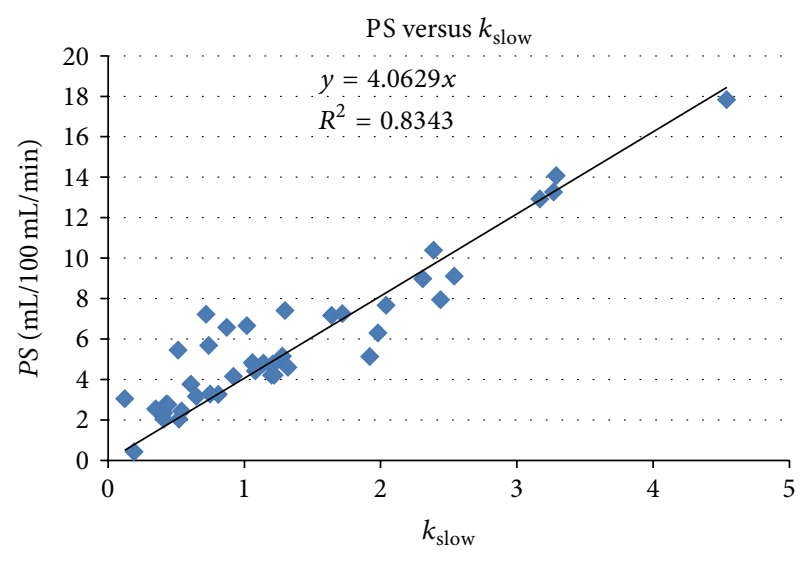

(b)

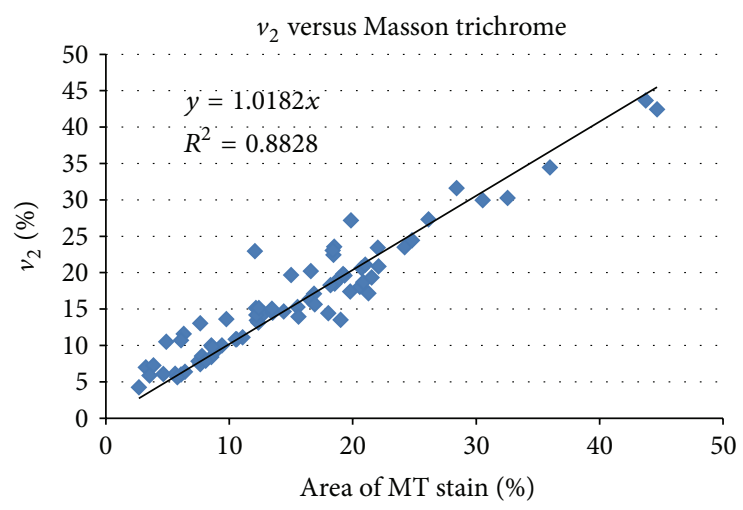

(d)

FIGURE 2: Correlation between (a) blood flow derived by the standard two-compartment model with the maximum slope of Galbumin uptake curve, (b) vascular permeability with $k_{\text {slow }}$ derived from Galbumin uptake curve, (c) intravascular space volume with \% of CD34 stain, and (d) interstitial space volume with \% of Masson trichrome stain.

Water PET has been used to validate plasma flow in DCECT. Bisdas et al. [20] used a distributed parameter (DP) model for tracer kinetic analysis in human brain studies to validate the derived perfusion values with $\mathrm{H}_{2}{ }^{15} \mathrm{O}$ PET scans and significant correlation was found with $r=0.50$ to 0.79 $\left(r^{2}\right.$-values ranged from 0.45 to 0.79$)$ and $0.01 \leq P \leq 0.05$. $\mathrm{H}_{2}{ }^{15} \mathrm{O}$-PET has also been used to validate tumor blood flow derived from DCE-MRI. Nevertheless, $K^{\text {trans }}$ was often used as a surrogate of blood flow and not $F$ directly [21]. Similarly, in another study using human melanoma xenografts, images of $E \cdot F$ (where $E$ is the extraction fraction) were obtained by subjecting DCE-MR images to Kety analysis and compared to $\mathrm{Na}^{99 \mathrm{~m}} \mathrm{TcO}_{4}$ as a perfusion tracer [22].

Unlike in DCE-CT, there has not been a study that validates $F$ estimated by DCE-MRI directly, as previous studies used $K_{\text {trans }}$ as a surrogate of blood flow. In this study we attempted to estimate vascular flow directly with a macromolecule Galbumin using initial slope method. 


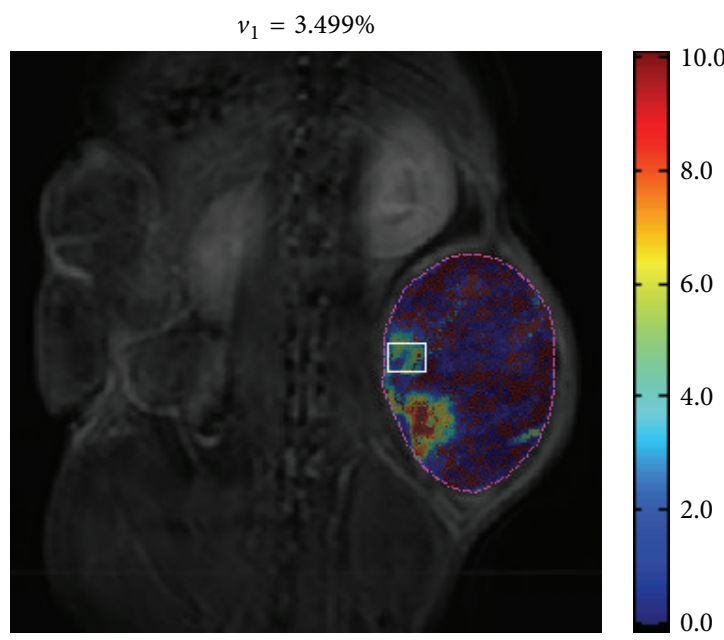

(a)

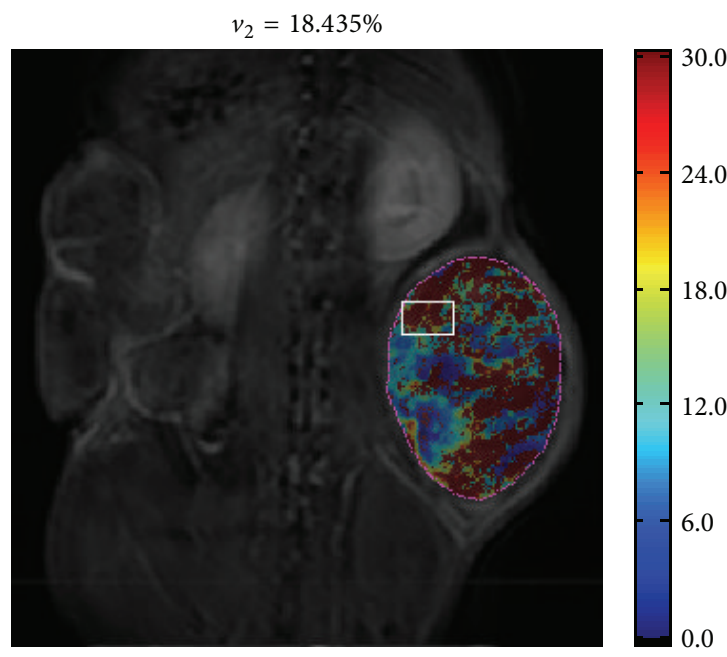

(d)

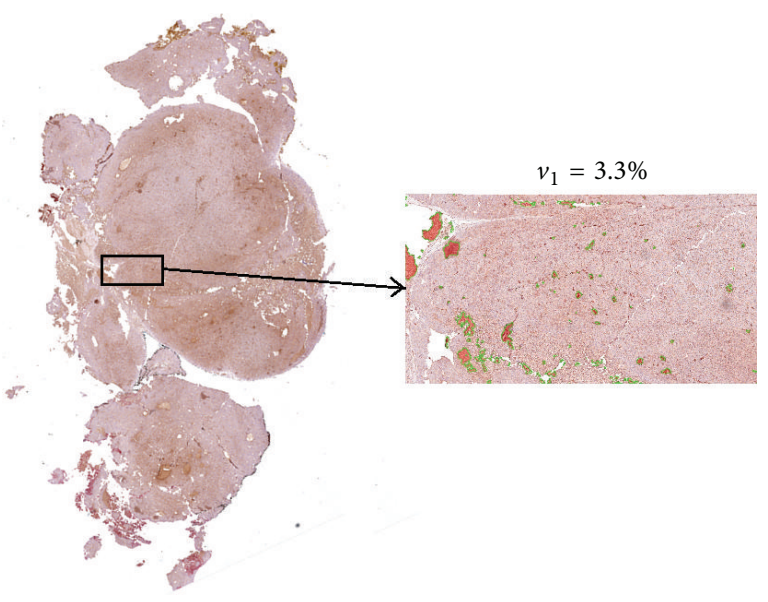

(b)

(c)

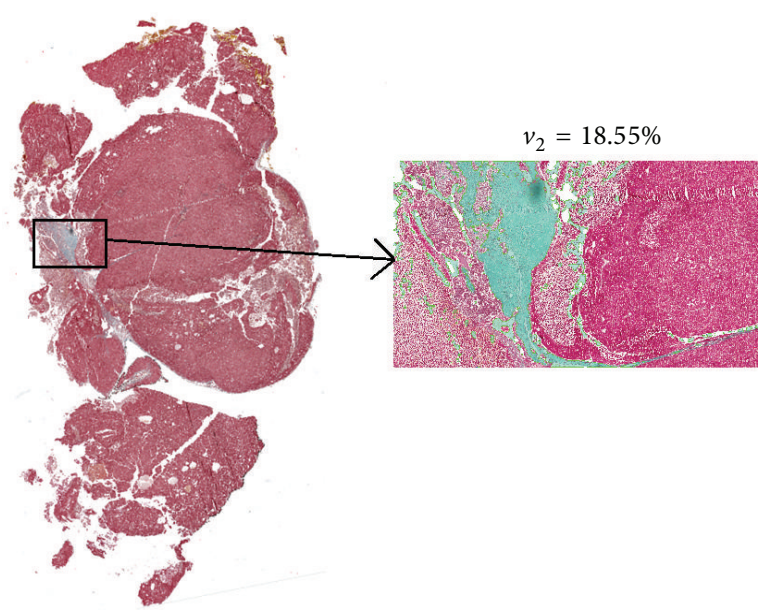

(e)

(f)

FIGURE 3: Fractional intravascular space volume of a mouse with HCC xenograft as derived by (a) the standard two-compartment model and (b) by the CD34 staining as shown in the inset of the whole tumor. The blood vessels are outlined in light green in (c) the hotspot. Fractional interstitial space volume of a mouse with HCC xenograft as derived by (d) the standard two-compartment model and (e) by the Masson trichrome staining as shown in the inset of the whole tumor. The interstitial space are outlined in light green in (f) the hotspot.

The good correlation between $F$ and vascular flow derived from the initial slope method indicates the feasibility of using $F$ as an estimate of vascular flow.

$K^{\text {trans }}$ has also been previously used to validate permeability. Ferrier et al. [1] used $K^{\text {trans }}$ as an estimate of vascular permeability and compared them to estimates $K$ obtained using $\left[{ }^{14} \mathrm{C}\right]$ aminoisobutyric acid quantitative autoradiography $\left(\left[{ }^{14} \mathrm{C}\right] \mathrm{AIB}\right.$ QAR), which was an established method of evaluating blood-tumor barrier permeability. Significant correlation was found between $K^{\text {trans }}$ and $K(r=0.905$, $P=0.035)$.

Nevertheless, it is more difficult to validate vascular permeability in general. The blood-to-brain influx rate constant $\left(K_{i}\right)$ as derived from the Patlak plot was the established estimate for vascular permeability [17]. The good correlation between PS and $K_{i}$ indicates the feasibility of using PS as an estimate of vascular permeability.

So, instead of using $K^{\text {trans }}$ which can mean either flow or permeability, in this study we have shown the ability to measure flow and permeability separately and validate them independently.

Egeland et al. [23] compared the fractional volume of the extravascular extracellular space (EESF) derived by DCEMRI with histology. They found that the numerical values of the DCE-MRI-derived parameters were not significantly different from the absolute values of tumor blood perfusion or fractional volume of the extravascular extracellular space in any of the tumor lines. Similarly, Benjaminsen et al. [24] investigated whether Gd-DTPA-based DCE-MRI can be used to assess the EESF of tumors using amelanotic human 


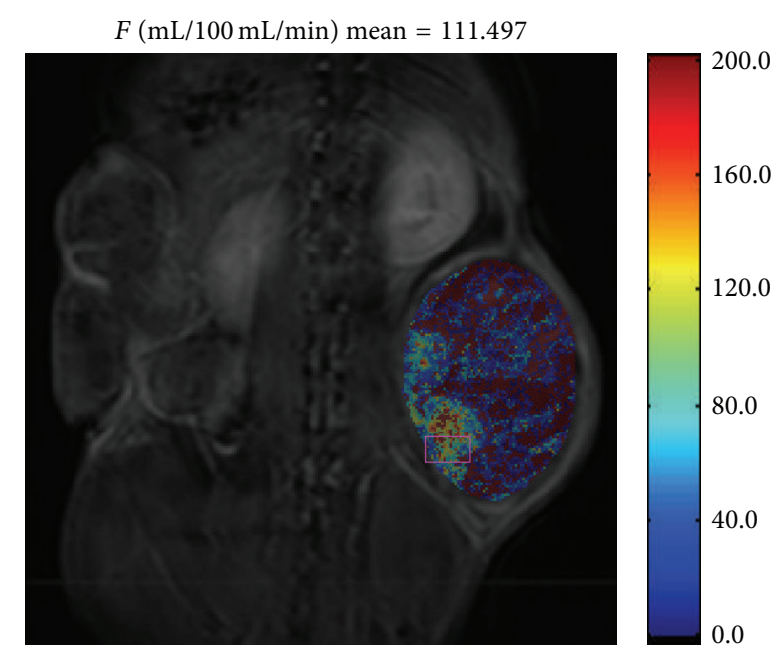

(a)

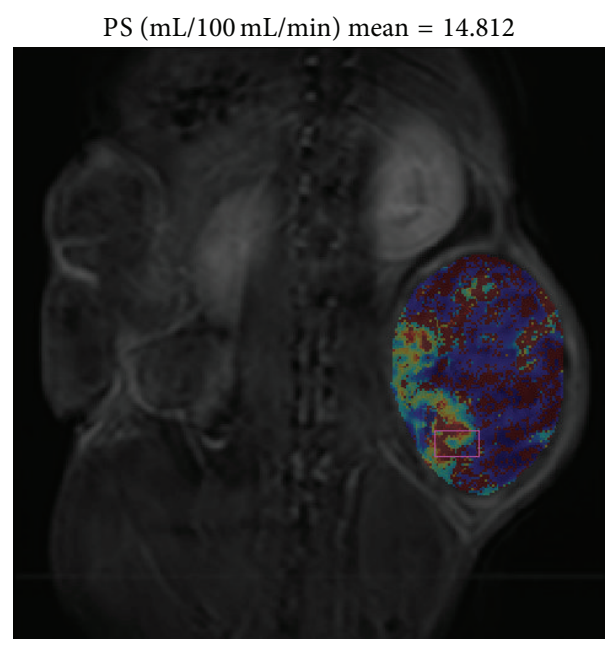

(c)

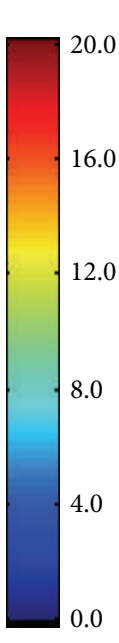

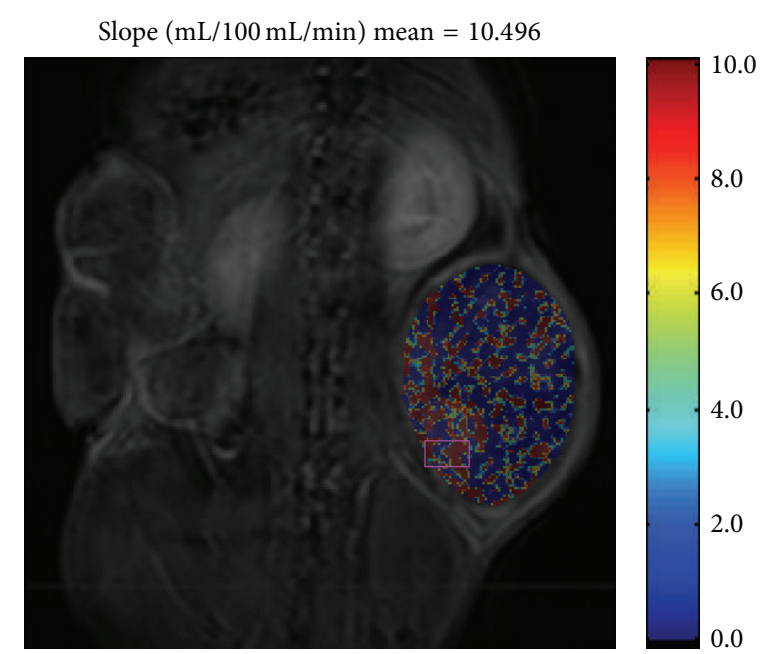

(b)

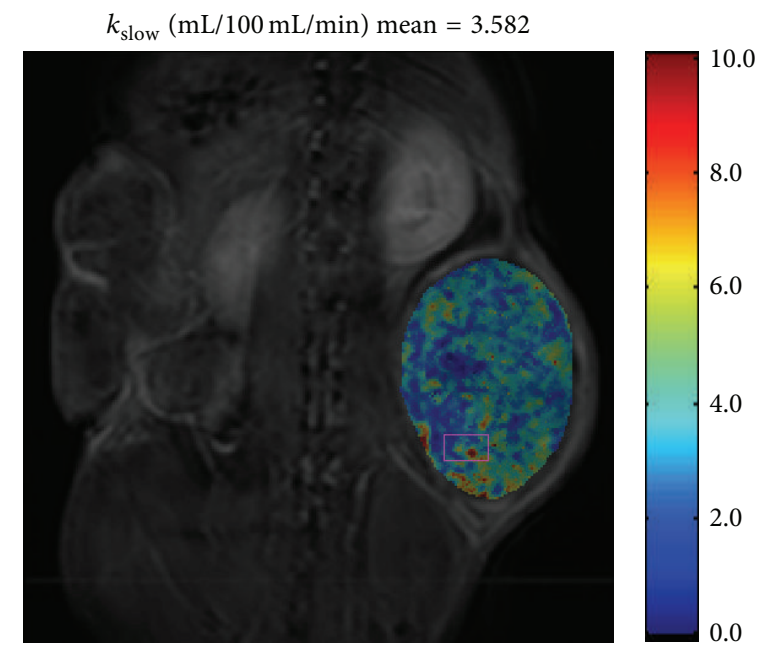

(d)

FIGURE 4: Blood flow of a mouse with HCC xenograft as derived by (a) the standard two-compartment model and (b) the slope of the Patlak plot. Vascular permeability of a mouse with HCC xenograft as derived by (c) the standard two-compartment model and (d) the $k_{\text {slow }}$ of the Patlak plot.

melanoma xenografts. Images of $\lambda$ (the partition coefficient of Gd-DTPA) were obtained by Kety analysis of DCE-MRI data. Positive correlations were found between $\lambda$ and EESF obtained by invasive imaging.

Gaustad et al. [25] investigated whether Gd-DTPA-based DCE-MRI can be a useful method for characterizing vascularity of tumors by comparing the images of $E \cdot F$ as obtained by Kety analysis with Blood Supply Time (BST) images (i.e., images of the time from when arterial blood enters a tumor through the supplying artery until it reaches a vessel segment within the tumor) and morphologic images of the microvascular network which were produced by intravital microscopy. They found that the $E \cdot F$ images mirrored the morphology (microvascular density) and the function (BST) of the microvascular networks well.

In this study, we compared both $v_{1}$ and $v_{2}$ directly with immunohistological chemistry stainings and not to invasive or morphological imaging.
This study suffered from the following limitations. As the parameters derived from DCE-MRI were compared with histology, there might be some distortions to the tissue during stain preparation. The orientation of scans might not exactly correspond with the histology slice as well. Difficulty in finding exact stains for flow and permeability also forced us to use a rather less than ideal substitute in the form of Galbumin (and its respective $K_{i}$ from Patlak plot).

\section{Conclusion}

We have shown that the parameters derived from the standard two-compartment model correlated with the respective values derived from histopathology.

It indicates the reliability of the proposed technique and potentials of applying this technique as a biomarker for preclinical drug developments, as it might allow monitoring of antiangiogenic therapy in murine models. 


\section{Conflict of Interests}

The authors would like to declare the following conflict of interests. Septian Hartono, Choon Hua Thng, and Tong San Koh received research funding from Roche-Singapore Translational Medicine Hub. Laurent Martarello is employed by Roche-Singapore Translational Medicine Hub.

\section{References}

[1] M. C. Ferrier, H. Sarin, S. H. Fung et al., "Validation of dynamic contrast-enhanced magnetic resonance imaging-derived vascular permeability measurements using quantitative autoradiography in the RG2 rat brain tumor model," Neoplasia, vol. 9, no. 7, pp. 546-555, 2007.

[2] C. A. Hulka, W. B. Edmister, B. L. Smith et al., "Dynamic echoplanar imaging of the breast: experience in diagnosing breast carcinoma and correlation with tumor angiogenesis," Radiology, vol. 205, no. 3, pp. 837-842, 1997.

[3] M. V. Knopp, E. Weiss, H. P. Sinn et al., "Pathophysiologic basis of contrast enhancement in breast tumors," Journal of Magnetic Resonance Imaging, vol. 10, no. 3, pp. 260-266, 1999.

[4] O. Ikeda, Y. Yamashita, and M. Takahashi, "Gd-enhanced dynamic magnetic resonance imaging of breast masses," Topics in Magnetic Resonance Imaging, vol. 10, no. 2, pp. 143-151, 1999.

[5] P. S. Tofts, G. Brix, D. L. Buckley et al., "Estimating kinetic parameters from dynamic contrast-enhanced t1-weighted MRI of a diffusable tracer: standardized quantities and symbols," Journal of Magnetic Resonance Imaging, vol. 10, no. 3, pp. 223232, 1999.

[6] S. Zwick, G. Brix, P. S. Tofts et al., "Simulation-based comparison of two approaches frequently used for dynamic contrastenhanced MRI," European Radiology, vol. 20, no. 2, pp. 432-442, 2010.

[7] P. Hayton, M. Brady, L. Tarassenko, and N. Moore, "Analysis of dynamic MR breast images using a model of contrast enhancement," Medical Image Analysis, vol. 1, no. 3, pp. 207-224, 1997.

[8] G. Brix, M. L. Bahner, U. Hoffman, A. Horvath, and W. Schreiber, "Regional blood flow, capillary permeability, and compartmental volumes: measurement with dynamic CTinitial experience," Radiology, vol. 210, no. 1, pp. 269-276, 1999.

[9] S. B. Donaldson, C. M. L. West, S. E. Davidson et al., "A comparison of tracer kinetic models for T1-weighted dynamic contrastenhanced MRI: application in carcinoma of the cervix," Magnetic Resonance in Medicine, vol. 63, no. 3, pp. 691-700, 2010.

[10] T. S. Koh, S. Bisdas, D. M. Koh, and C. H. Thng, "Fundamentals of tracer kinetics for dynamic contrast-enhanced MRI," Journal of Magnetic Resonance Imaging, vol. 34, no. 6, pp. 1262-1276, 2011.

[11] C. S. Patlak, R. G. Blasberg, and J. D. Fenstermacher, "Graphical evaluation of blood-to-brain transfer constants from multiple-time uptake data," Journal of Cerebral Blood Flow and Metabolism, vol. 3, no. 1, pp. 1-7, 1983.

[12] H. Huynh, K. C. Soo, P. K. H. Chow, L. Panasci, and E. Tran, "Xenografts of human hepatocellular carcinoma: a useful model for testing drugs," Clinical Cancer Research, vol. 12, no. 14, pp. 4306-4314, 2006.

[13] H. Z. Wang, S. J. Riederer, and J. N. Lee, "Optimizing the precision in T1 relaxation estimation using limited flip angles," Magnetic Resonance in Medicine, vol. 5, no. 5, pp. 399-416, 1987.
[14] J. A. Brookes, T. W. Redpath, F. J. Gilbert, G. Needham, and A. D. Murray, "Measurement of spin-lattice relaxation times with FLASH for dynamic MRI of the breast," The British Journal of Radiology, vol. 69, no. 819, pp. 206-214, 1996.

[15] R. G. Tilton, K. B. Larson, J. R. Udell, B. E. Sobel, and J. R. Williamson, "External detection of early microvascular dysfunction after no-flow ischemia followed by reperfusion in isolated rabbit hearts," Circulation Research, vol. 52, no. 2, pp. 210-225, 1983.

[16] K. A. Miles, "Perfusion imaging with computed tomography: brain and beyond," European Radiology, vol. 16, supplement 7, pp. M37-M43, 2006.

[17] T. N. Nagaraja, K. Karki, J. R. Ewing et al., “The MRI-measured arterial input function resulting from a bolus injection of GdDTPA in a rat model of stroke slightly underestimates that of Gd- $\left[{ }^{14} \mathrm{C}\right] \mathrm{DTPA}$ and marginally overestimates the blood-tobrain influx rate constant determined by Patlak plots," Magnetic Resonance in Medicine, vol. 63, no. 6, pp. 1502-1509, 2010.

[18] M. Aref, A. R. Chaudhari, K. L. Bailey, S. Aref, and E. C. Wiener, "Comparison of tumor histology to dynamic contrast enhanced magnetic resonance imaging-based physiological estimates," Magnetic Resonance Imaging, vol. 26, no. 9, pp. 1279-1293, 2008.

[19] E. E. Stewart, X. Chen, J. Hadway, and T.-Y. Lee, "Hepatic perfusion in a tumor model using DCE-CT: an accuracy and precision study," Physics in Medicine \& Biology, vol. 53, no. 16, pp. 4249-4267, 2008.

[20] S. Bisdas, F. Donnerstag, G. Berding, T. J. Vogl, C. H. Thng, and T. S. Koh, "Computed tomography assessment of cerebral perfusion using a distributed parameter tracer kinetics model: validation with $\mathrm{H}_{2}^{(15)} \mathrm{O}$ positron emission tomography measurements and initial clinical experience in patients with acute stroke," Journal of Cerebral Blood Flow and Metabolism, vol. 28, no. 2, pp. 402-411, 2008.

[21] A. J. De Langen, M. Lubberink, R. Boellaard et al., "Reproducibility of tumor perfusion measurements using ${ }^{15} \mathrm{O}$-labeled water and PET," Journal of Nuclear Medicine, vol. 49, no. 11, pp. 1763-1768, 2008.

[22] I. C. Benjaminsen, B. A. Graff, K. G. Brurberg, and E. K. Rofstad, "Assessment of tumor blood perfusion by high-resolution dynamic contrast-enhance MRI: a preclinical study of human melanoma xenografts," Magnetic Resonance in Medicine, vol. 52, no. 2, pp. 269-276, 2004.

[23] T. A. M. Egeland, T. G. Simonsen, J.-V. Gaustad, K. Gulliksrud, C. Ellingsen, and E. K. Rofstad, "Dynamic contrast-enhanced magnetic resonance imaging of tumors: preclinical validation of parametric images," Radiation Research, vol. 172, no. 3, pp. 339-347, 2009.

[24] I. C. Benjaminsen, K. G. Brurberg, E.-B. M. Ruud, and E. K. Rofstad, "Assessment of extravascular extracellular space fraction in human melanoma xenografts by DCE-MRI and kinetic modeling," Magnetic Resonance Imaging, vol. 26, no. 2, pp. 160-170, 2008.

[25] J. V. Gaustad, K. G. Brurberg, T. G. Simonsen, C. S. Mollatt, and E. K. Rofstad, "Tumor vascularity assessed by magnetic resonance imaging and intravital microscopy imaging," Neoplasia, vol. 10, no. 4, pp. 354-362, 2008. 


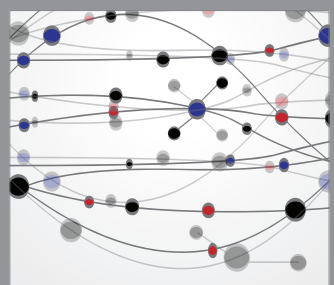

The Scientific World Journal
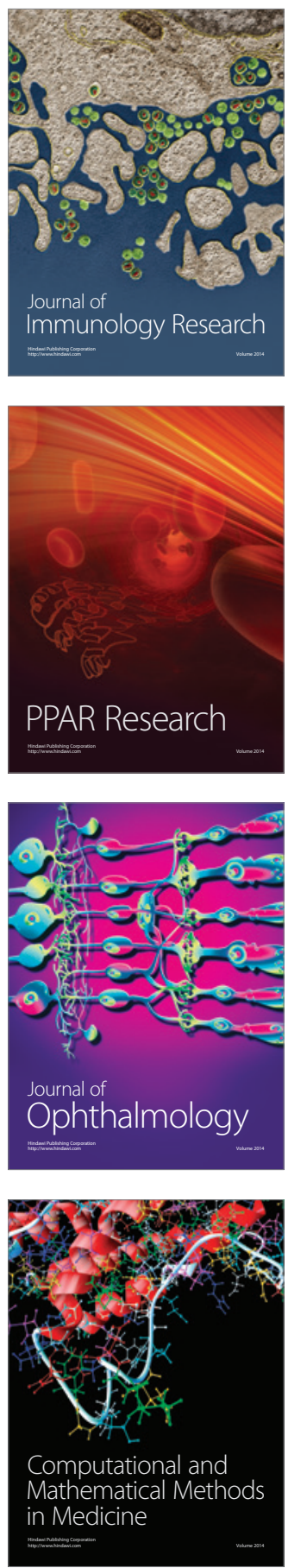

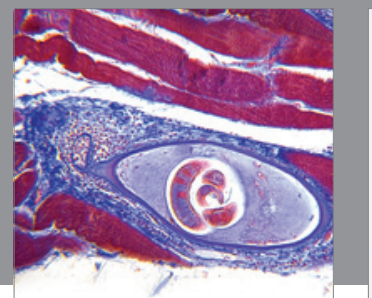

Gastroenterology

Research and Practice
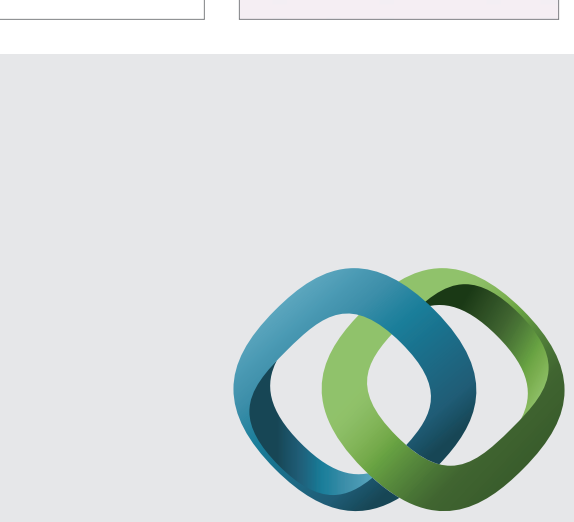

\section{Hindawi}

Submit your manuscripts at

http://www.hindawi.com
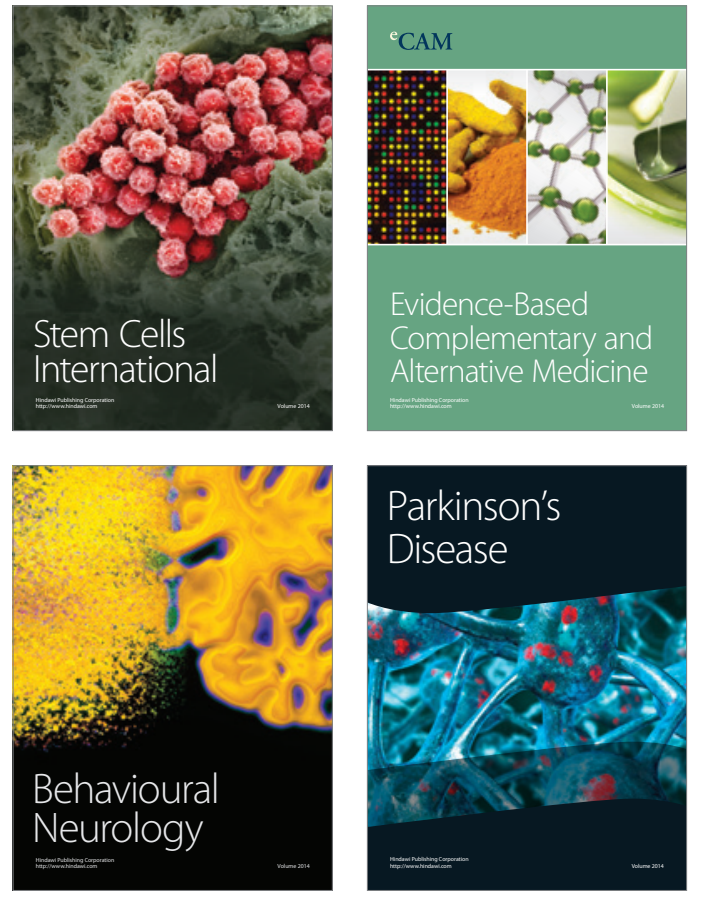
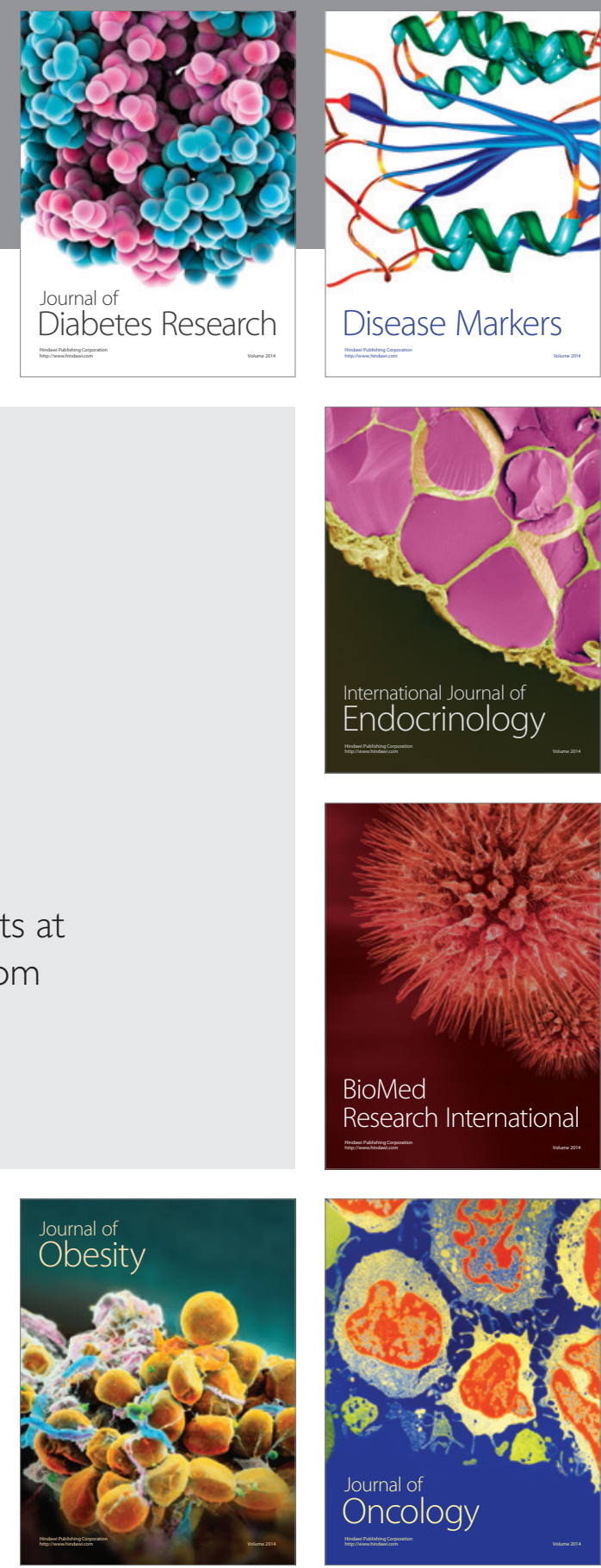

Disease Markers
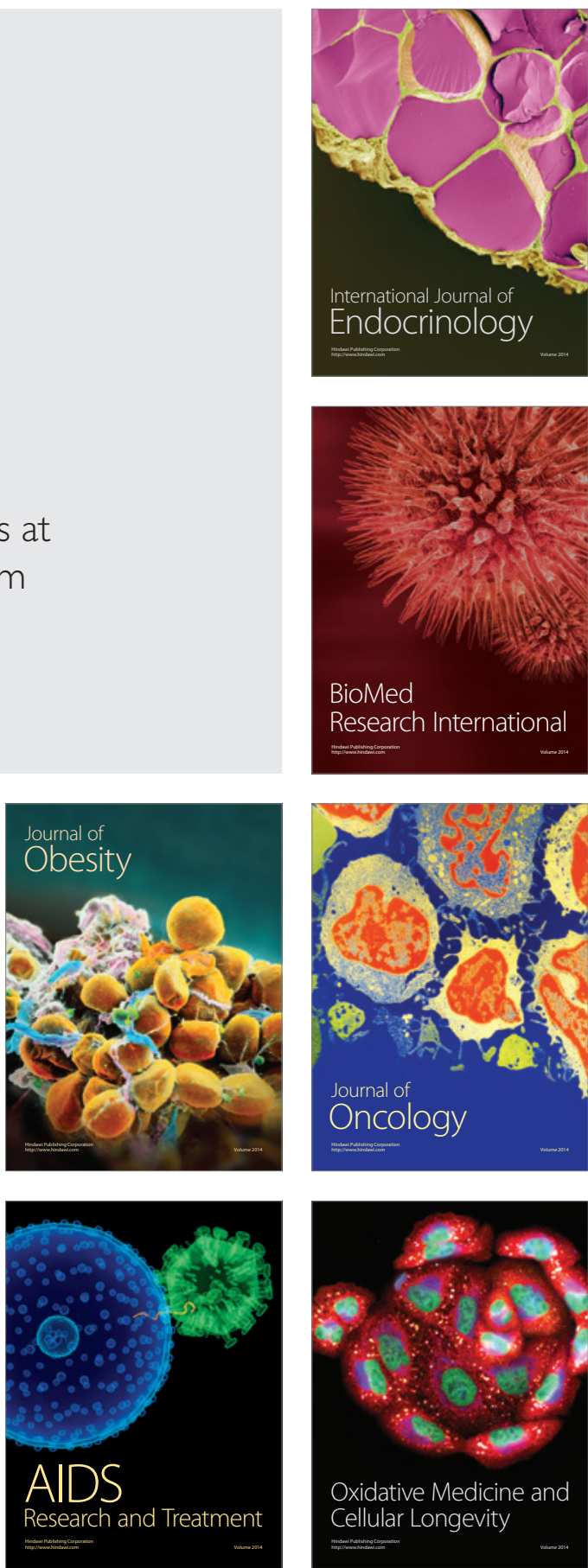